\title{
1 Phytotoxic effects of volatile and water soluble chemicals of
}

4 ANTONIO I. ARROYO ${ }^{*}$, YOLANDA PUEYO ${ }^{1}$, FRANÇOIS PELLISSIER $^{2}$, JAVIER $^{2}$ RAMOS $^{3}$, ANA ESPINOSA-RUIZ ${ }^{4}$, ANNIE MILLERY² AND CONCEPCIÓN L.

$\operatorname{ALADOS}^{1}$

7

$8{ }^{1}$ Instituto Pirenaico de Ecología, Consejo Superior de Investigaciones Científicas, Av. Montañana 1005, 50059 Zaragoza, Spain.

${ }^{2}$ Université Savoie Mont Blanc, CNRS, LECA, F-73000 Chambéry, France.

${ }^{3}$ Estación Experimental Aula Dei, Consejo Superior de Investigaciones Científicas, Av. Montañana 1005, 50059 Zaragoza, Spain.

14 Valencia-Consejo Superior de Investigaciones Científicas, C/ ingeniero Fausto Elio s/n, 46022 Valencia, Spain. 


\section{Abstract}

20

21

22

We investigated the phytotoxic effects of volatile and water soluble chemicals produced by the shrub Artemisia herba-alba Asso. We conducted a number of germination and early seedling growth bioassays on species that co-exist with A. herba-alba in natural semiarid plant communities (Salsola vermiculata L., Lygeum spartum L., Pinus halepensis Mill. and A. herba-alba itself). In addition, we assessed the phytotoxic effects of a mixture of phenols that were identified in the aqueous extract of $A$. herbaalba on the germination of such species. We found that volatile chemicals inhibited the germination of $P$. halepensis seeds, promoted the growth of $P$. halepensis seedlings and reduced the root biomass of $S$. vermiculata seedlings, while water soluble chemicals promoted the growth of L. spartum and P. halepensis seedlings. On the other hand, both volatile and water soluble compounds inhibited the germination of $A$. herba-alba seeds, highlighting its strong autotoxic nature. We did not find a phytotoxic effect of phenols identified (catechol, protocatechuic and vanillic acids) on species that co-exists with $A$. herba-alba, although they had a moderate autotoxic effect. Our results suggest that both the volatile and water soluble chemicals might be involved in chemical interactions among plants in semiarid environments.

\section{Key Words}

Aqueous extract, Germination, Phenolic compounds, Seedling growth, Semiarid environment, Volatile compounds. 


\section{Introduction}

41 Many plants species interfere with neighboring plants beyond direct competition for resources, through the production and release into the environment of secondary metabolites (allelochemicals) by leachates, litter decomposition, root exudates and volatilization (Rice, 1984). Allelochemicals can affect other plants directly by reducing seed germination, growth and survival (Rice, 1984) and indirectly by altering soil microbial communities and their effects on soil biogeochemical processes, e.g., nitrification (Castaldi et al., 2009; Wang et al., 2012). However, allelochemicals are often non-specific compounds and allelopathy may also occur within the same species, which is known as autotoxicity (Ruan et al., 2011). Thus, autotoxicity occurs when allelochemicals released by a plant negatively interfere with germination, growth and survival of the same plant species (Friedman and Waller, 1985; Singh et al., 1999). As such, plant-plant interactions mediated by allelochemicals (i.e., allelopathy and autotoxicity) have the potential to shape the composition and structure of the aboveground vegetation (Alías et al., 2006; Herranz et al., 2006).

Many species are known to be potentially allelopathic in semiarid environments (Araniti et al., 2012; Friedjung et al., 2013). Indeed, harsh abiotic factors such as high temperatures, intense solar radiation and water deficit often enhance the production, accumulation and phytotoxicity of allelochemicals (Akula and Ravishankar, 2011; Chen et al., 2012). Furthermore, in those communities, the joint action of multiple stresses might increase the plant's susceptibility to the effects of allelochemicals (Pedrol et al., 2006). It has been argued that allelopathy may be the result of selection against being facilitative plants (van der Putten, 2009). Similarly, autotoxicity can play a relevant role in avoiding the impacts of intraspecific competition for scarce resources (e.g., water) 
under harsh environmental conditions (Armas and Pugnaire, 2011). However, autotoxicity has been rarely explored despite its potential adaptive value in those systems.

It has been claimed that plants in arid areas release mostly volatile

allelochemicals (e.g., monoterpenes) while water soluble allelochemicals (e.g., phenols) are more common in cool temperate areas (Chou, 1999; Reigosa et al., 1999). In semiarid environments, however, plants are especially rich in phenols because droughttolerant plants produce and accumulate those compounds to overcome the oxidative damage caused by drought stress (Bautista et al., 2016; Varela et al., 2016). In addition, even though water scarcity might act as a physical limitation to diffusion of water soluble chemicals, in these systems, there is a substantial water redistribution from the bare soil to vegetation patches and other mechanisms (e.g., water input from fog drip; Callaway, 2007; Ludwig et al., 2005) that may increase the water soluble allelochemicals available to vegetation patches. Consequently, the role of water soluble chemicals, and in particular phenols, on allelopathy and autotoxicity in semiarid environments might have been overlooked.

Phenolic compounds are one of the best-known classes of plant allelochemicals (Muscolo and Sidari, 2010). Phenols comprise several groups including simple phenols, phenolic acids, coumarins, tannins and some flavonoids (Li et al., 2010; Muscolo and Sidari, 2010). Mainly, they are introduced into the environment through plant litter decomposition and, to a lesser extent, as leachates of plant parts (Hättenschwiler and Vitousek, 2000). Once the phenols are released, they interact with nutrient cycling and can limit the availability of certain nutrients (e.g., nitrogen and phosphate;

Hättenschwiler and Vitousek, 2000; Inderjit and Mallik, 1997). Biological effects of phenolic compounds in target plants range from changes in cell membrane permeability 
to effects on plant photosynthesis, which affect the normal growth and development of the entire plant (for an extensive review, see Li et al., 2010).

The objective of this study was to investigate the phytotoxic effects of the volatile and water soluble chemicals produced by an allelopathic shrub of a semiarid plant community. As allelopathic species we selected Artemisia herba-alba Asso. (desert wormwood; Mohamed et al., 2010), a dwarf shrub about 30-40 cm tall, widespread in semiarid areas of the Mediterranean Basin, especially in the Iberian Peninsula, North Africa and the Middle East. Aerial parts of A. herba-alba, including leaves, young stems and flowers, contain high amounts of phenolic compounds (Bourgou et al., 2016; Khlifi et al., 2013; Younsi et al., 2016). However, although the effects of A. herba-alba aqueous extract have been assessed on different target species (Arroyo et al., 2016; Atoum et al., 2006), the phenolic constituents of the aqueous extract seldom have been characterized. On the other hand, the composition of the essential oils of A. herba-alba has been largely investigated (Mohamed et al., 2010; Salido et al., 2004; Younsi et al., 2016), although its phytotoxicity through volatilization has not been assessed before (but see Friedman et al., 1977). We hypothesized that i) not only volatile, but also water soluble chemicals produced by A. herba-alba have phytotoxic effects on seed germination and early seedling growth. We further hypothesized that ii) aqueous extract of A. herba-alba is rich in phenolic compounds and that iii) some of those phenolic compounds (i.e., identified ones) induce phytotoxic activity.

To address the first hypothesis, we conducted germination and growth bioassays to assess phytotoxic effects of volatile and water soluble chemicals on a set of target species. Target species included the shrub Salsola vermiculata L., the perennial grass Lygeum spartum L. (two dominant species that co-exist with A. herba-alba in natural 
semiarid plant communities) and Pinus halepensis Mill. (a species commonly used to reforest areas that have natural populations of A. herba-alba). Artemisia herba-alba was also included as target species to test for autotoxicity (Atoum et al., 2006; Friedman and Orshan, 1975). To address the second and third hypotheses, we first quantified the Total Phenolic Content (TPC) of the aqueous extract, and of the leaves and stems of $A$. herba-alba to estimate the fraction that is actually water soluble. Later, some phenolic compounds in the aqueous extract were identified based on High Performance Liquid Chromatography (HPLC), and the potential phytotoxic effects of the phenolic compounds identified were assessed on seed germination of target species. Additionally, the volatile organic compounds (VOCs) in the shoots of $A$. herba-alba were also identified based on Gas Chromatography-Mass Spectrometry (GC-MS).

\section{Material and Methods}

\subsection{Plant Material Collection and Aqueous Extraction}

Mature seeds of A. herba-alba and S. vermiculata, and mature caryopses of L. spartum were collected in the field, in 2014, from a natural plant community in the Middle Ebro Valley, NE Spain. This area is characterized by a semiarid climate, and has an average annual precipitation that ranges between 300 and $400 \mathrm{~mm}_{\text {year }}{ }^{-1}$. Mean annual temperature is about $15^{\circ} \mathrm{C}$, with a pronounced continentality (data obtained from the Digital Climatic Atlas of Aragón; http://anciles.aragon.es/AtlasClimatico/). Seeds of $P$. halepensis, which were collected in 2011, were obtained from a local garden center. Furthermore, aerial parts (i.e., leaves and stems) of $A$. herba-alba were collected from a number of individuals, randomly selected, in the same plant community before the beginning of bioassays. Specifically, aerial parts of $A$. herba-alba used in the aqueous extraction and in the TPC analysis were collected in March 2015, and aerial parts used 
in the volatile bioassays and in the VOCs analysis were collected in December 2015.

Aerial parts were air-dried at room temperature for $10 \mathrm{~d}$ and stored in a cold chamber at $4^{\circ} \mathrm{C}$ until its utilization. In the drying process, fresh material lost more than $40 \%$ of its weight. The dried aerial parts used in the bioassays of the volatile chemicals were ground nearly to powder, by a mechanical mill, prior to the start of the bioassays.

To obtain the aqueous extract of A. herba-alba, the dried aerial parts were soaked in demineralized water $\left(100 \mathrm{~g} \mathrm{l}^{-1}\right)$ for $24 \mathrm{~h}$ at room temperature $\left(15^{\circ} \mathrm{C}-20^{\circ} \mathrm{C}\right)$ in total darkness. The solution was filtered, stored in small portions $(30 \mathrm{ml})$ and frozen ($18^{\circ} \mathrm{C}$ ) until its utilization.

\subsection{Determination of the Phytotoxic Effects of Volatile and Water Soluble}

\section{Chemicals}

\subsubsection{Seed Germination Bioassays}

The phytotoxic effect of the volatile chemicals released from the aerial parts of $A$. herba-alba was determined by assessing seed germination in the four target species $(S$. vermiculata, L. spartum, P. halepensis and A. herba-alba). Seeds were placed on a layer of Joseph filter paper within 10-cm-diameter Petri dishes and were moistened with $5 \mathrm{ml}$ of demineralized water. Aluminum foil containers (3-cm-diameter) with $0.05,0.1$ or 0.5 $\mathrm{g}$ (dry weight) of ground aerial parts were then placed into Petri dishes. Ten replicates (i.e., Petri dishes) of S. vermiculata, P. halepensis and A. herba-alba (10 seed each), and L. spartum (5 caryopses each) were utilized at each treatment. The controls were performed placing empty aluminum containers within Petri dishes. Petri dishes were hermetically sealed with parafilm. Seeds were set to germinate in a greenhouse under controlled conditions ( $12 \mathrm{~h}$ of light at $23^{\circ} \mathrm{C}$ and $12 \mathrm{~h}$ of darkness at $18^{\circ} \mathrm{C}$; Table A.1). 

assessing seed germination in the four target species following the same design mentioned above (Table A.1). In this case, seeds were moistened with $5 \mathrm{ml}$ of aqueous extract diluted to $0.5,2$ or $5 \mathrm{~g}^{-1}$, and controls were performed leaching seeds with demineralized water. In addition, seeds were set to germinate in a room that had a temperature that ranged between $24^{\circ} \mathrm{C}$ in the day and $20^{\circ} \mathrm{C}$ at night, and $12 \mathrm{~h}$ of light provided by cool light fluorescent tubes $\left(204 \mu \mathrm{mol} \mathrm{s}{ }^{-1} \mathrm{~m}^{-2}\right)$. Germination of $A$. herbaalba seeds failed in those conditions and required lower temperatures to succeed. that had $12 \mathrm{~h}$ of light photoperiod $\left(210 \mu \mathrm{mol} \mathrm{s} \mathrm{m}^{-1}\right)$, light period temperature $15^{\circ} \mathrm{C}$ and dark period temperature $5^{\circ} \mathrm{C}$ (Table A.1). The positions of the Petri dishes were changed randomly every few days and the number of germinated seeds (rupture of seed coats and protrusion of the radicle) was recorded daily for $21 \mathrm{~d}$.

The phytotoxic effect of a mixture of pure phenolic compounds was also determined by assessing seed germination in the four target species. Specifically, catechol, protocatechuic and vanillic acids were used for the mixture because they were identified and quantified in A. herba-alba aqueous extract (40.6 $\mathrm{mg} \mathrm{l}^{-1}$ in $100 \mathrm{~g}^{-1}$ aqueous extract; 37.2, 9.4 and $2.2 \mathrm{mg} \mathrm{l}^{-1}$ respectively). Seeds were moistened with $5 \mathrm{ml}$ of three dilutions of the phenolic mixture as treatments $\left(0.24,0.98\right.$ and $\left.2.44 \mathrm{mg} \mathrm{l}^{-1}\right)$. The proportion found for each identified compound was maintained when the mixture was performed. Thus, at each treatment, the concentration of each compound was the same as the concentration of such compounds in either $0.5,2$ or $5 \mathrm{~g} \mathrm{l}^{-1}$ of A. herba-alba aqueous extract. Seeds were moistened with demineralized water in the controls. The number of replicates, number of seeds per treatment and germination conditions was the same as those in the germination bioassay of aqueous extract (Table A.1). 


\subsubsection{Early seedling growth bioassays}

Seeds of S. vermiculata, L. spartum, P. halepensis and A. herba-alba were sowed in $8 \mathrm{x}$ $8 \times 7 \mathrm{~cm}$ plastic pots (60 pots per species and bioassay) that were filled with approximately $100 \mathrm{~g}$ of substrate (peat). To maximize the probability of having at least one seedling per pot, multiple seeds were sowed in each pot. Extra seedlings were removed once more than one seedling had emerged in a pot. Exceptionally, two seedlings of $P$. halepensis were left to grow in some pots because germination was low. Pinus halepensis seedlings have pivoting roots, with limited lateral development (personal observation) and, consequently, neighboring seedlings had negligible influence. Pot positions were randomized in space when seeds were set to germinate. In the germination period, pots were watered regularly with tap water. After most of the seedlings had become well established (approximately two weeks), treatments were applied.

The phytotoxic effect of the volatile chemicals released from aerial parts of $A$. herba-alba was determined by assessing early seedling growth in the four target species. Seedlings were set to grow in three portable plastic greenhouses $\left(0.29 \mathrm{~m}^{3}\right)$ under a $12 \mathrm{~h}$ of natural daylight photoperiod and a temperature that ranged between $20^{\circ} \mathrm{C}$ and $25^{\circ} \mathrm{C}$ on clear days (temperature did not differ significantly among greenhouses, $F_{3,24}=0.061, p>0.05$, data not shown). Fifteen pots of each seedling species were randomly placed into each greenhouse (60 pots per greenhouse) and either, 3,10 or $30 \mathrm{~g}$ of ground aerial parts (equivalent to $0.05,0.17$ or $0.5 \mathrm{~g}$ per pot respectively) were placed in a plastic support that hung about $20 \mathrm{~cm}$ above the pots. Artemisia herba-alba aerial parts were replaced twice in the bioassay. Controls pots (n $=60$ ) were randomly placed into a fourth greenhouse without the aerial parts of $A$. herba-alba (Table A.1). The positions of the portable greenhouses and pots were 
randomly changed every few days. Pots were watered regularly with tap water.

Greenhouses were open for about 10 min when pots were watered.

The phytotoxic effect of the water soluble chemicals of A. herba-alba was determined by assessing early seedling growth in the four target species. Specifically, 15 pots per species were watered weekly with $40 \mathrm{ml}$ of aqueous extract diluted to either, $0.5,2$ or $5 \mathrm{~g} \mathrm{l}^{-1}$. Occasionally, the treatment was reduced to $30 \mathrm{ml}$ because the substrate was nearly saturated. In those instances, to ensure that the dosages of water soluble chemicals were maintained, the concentrations of the solutions were increased to 0.7 , 2.7 and $6.7 \mathrm{~g} \mathrm{l}^{-1}$ respectively. Irrigation with tap water remained as control for the rest of pots. All pots were set to grow in a room that had a temperature of $24^{\circ} \mathrm{C}$ in the day and $20^{\circ} \mathrm{C}$ at night, and 12 hours of light provided by cool light fluorescent tubes (204 $\mu$ mol $\mathrm{s}^{-1} \mathrm{~m}^{-2}$; Table A.1). The positions of the pots were changed randomly every few days. After eight weeks growing, seedlings were dried at $60{ }^{\circ} \mathrm{C}$ for 3-4 days, and the biomass of the whole aerial parts and roots of the seedlings was measured.

\subsection{Determination of Volatile and Phenolic constituents}

\subsubsection{VOCs Extraction and GC-MS Analysis}

A 7.5-mg sample of powdered aerial parts was resuspended in $1 \mathrm{ml}$ of distilled water and sonicated for 5 minutes. Then, $0.1 \mathrm{ml}$ of the aqueous extract was placed into a $22 \mathrm{ml}$ crimp cap headspace vial and subjected to headspace solid phase microextraction (HSSPME). A 65 uM PDMS/DVB (Supelco, Bellefonte, PA, USA) fiber was used for the analysis. Pre-incubation and extraction times were performed at $50{ }^{\circ} \mathrm{C}$ for 10 and 20 min respectively. Desorption was performed for $1 \mathrm{~min}$ at $250^{\circ} \mathrm{C}$ in splitless mode. VOCs trapped on the fiber were analyzed by GC-MS using an autosampler COMBI PAL CTC Analytics (Zwingen, Switzerland), a 6890N GC Agilent Technologies (Santa 
Clara, CA, USA) and a 5975B Inert XL MSD Agilent, equipped with an Agilent J\&W

Scientific DB-5 fused silica capillary column (5\%-phenyl-95\%-dimethylpolysiloxane as stationary phase, $60 \mathrm{~m}$ length, $0.25 \mathrm{~mm}$ i.d., and $1 \mathrm{~mm}$ thickness film). Oven temperature conditions were $40{ }^{\circ} \mathrm{C}$ for $2 \mathrm{~min}, 5^{\circ} \mathrm{C} \min ^{-1}$ ramp until $250{ }^{\circ} \mathrm{C}$ and then held isothermally at $250{ }^{\circ} \mathrm{C}$ for $5 \mathrm{~min}$. Helium was used as carrier gas at $1.4 \mathrm{ml} \mathrm{min}{ }^{-1}$ constant flow. Mass/z detection was obtained by an Agilent mass spectrometer operating in the EI mode (ionization energy, $70 \mathrm{eV}$; source temperature $230{ }^{\circ} \mathrm{C}$ ). Data acquisition was performed in scanning mode (mass range $\mathrm{m} / \mathrm{z} 35-220$ ). Chromatograms and spectra were recorded and processed using the Enhanced ChemStation software for GC-MS (Agilent). Compound identification was based on both the comparison between the MS for each putative compound with those of the NIST 2005 Mass Spectral library and the match to a GC retention time and Mass Spectra custom library generated using commercially available compounds.

\subsubsection{Total Phenolic Content and HPLC-UV Analysis}

Total Phenolic Content (TPC) of the aqueous extract, leaves and stems of $A$. herba-alba was measured by spectrophotometry following the Folin-Ciocalteu method (Singleton and Rossi, 1965). Three 0.1-g samples of powdered leaves and stems from various individuals were extracted with $50 \mathrm{ml}$ of $70 \%$ ethanol and heated at ebullition temperature for $30 \mathrm{~min}$. Ethanol extracts were filtered (filtration funnel with filter $\mathrm{n}^{\mathbf{o}} 2$, 40-100 $\mu \mathrm{m}$ ), and $0.1 \mathrm{ml}$ of each extract was diluted in $3.4 \mathrm{ml}$ of distilled water before $0.5 \mathrm{ml}$ of Folin-Ciocalteu reagent was added. After $1 \mathrm{~min}$ incubation, $1 \mathrm{ml}$ of $\mathrm{Na}_{2} \mathrm{CO}_{3}$ solution (20\%) was added. The resultant solution was mixed thoroughly by mechanical shaking before being heated in a bain-marie at $40{ }^{\circ} \mathrm{C}$ for $20 \mathrm{~min}$. On the other hand, three 0.02-ml samples of aqueous extract of $A$. herba-alba were diluted in $3.48 \mathrm{ml}$ distilled water, and the same above-mentioned protocol was followed. The absorbance 
of the samples was read by spectrophotometry at $760 \mathrm{~nm}$ (Shimadzu UV-160) against the blank test (distilled water). TPC of the samples was estimated based on a calibration curve that was prepared with standard gallic acid ( 1 to $\left.10 \mu \mathrm{g} \mathrm{ml}^{-1}\right)$ and was expressed as mg of gallic acid equivalents (GAE) per g of plant material.

Phenolic compounds of the aqueous extract of $A$. herba-alba were identified by HPLC. Before the chemical analysis, an acid hydrolysis was performed to obtain free phenolic compounds. Specifically, $0.3 \mathrm{ml}$ of concentrated hydrochloric acid was added to $1.5 \mathrm{ml}$ aqueous extract of $A$. herba-alba in a glass tube, which was heated at $100{ }^{\circ} \mathrm{C}$ for $1 \mathrm{~h}$. This solution was filtered through a 1- $\mu \mathrm{m}$ glass-microfiber disc before being injected into the HPLC system. A pre-column Waters $\mu$ Bondapak C18 Sentry Guard Cartridge, $(10 \mu \mathrm{m}, 3.9 \mathrm{~mm} \times 20 \mathrm{~mm})$ was placed immediately before a Waters column $\mu$ Bondapak $18 \mathrm{C}$ (250 mm x $4.6 \mathrm{~mm}$ ID cartridge, $10 \mu \mathrm{m})$. The phenolic compounds were eluated with a flow rate of $1.5 \mathrm{ml} \mathrm{min}^{-1}$ and a linear gradient from 0 to $20 \%$ of solvent B (acetonitrile) in solvent A (distilled water with $0.5 \%$ glacial acetic acid), followed by 15 min re-equilibration with solvent $\mathrm{A}$. The UV-visible spectrum was recorded at $280 \mathrm{~nm}$ (maximum absorbance of phenolic compounds) with a Waters 996 photodiode array detector. The compounds were identified and quantified by comparing the retention time, the absorption spectrum and the peak area to those of a standard.

\subsection{Statistical Analyses}

Differences in seed germination among treatments were analyzed, for each target species, using generalized linear mixed models (GLMMs) for repeated measures. Treatment and time of measurement were set as fixed factors, while Petri dish was included as random factor. Three moments were considered as time of measurement: 7 , 14 and 21 days (d7, d14 and $\mathrm{d} 21$ respectively) after the start of the germination 
bioassays (except $S$. vermiculata because germination had ended by d14). A binomial distribution of errors was specified in the models. Tukey's post hoc honest significant difference (HSD) tests were used to detect significant differences between pairs of treatments.

Differences in the aerial and root biomass of seedlings among treatments in the growth bioassays were evaluated, for each target species, using one-way ANOVA. Tukey's post hoc honest significant difference (HSD) tests were used to detect significant differences between pairs of treatments.

$$
\text { Statistical analyses were performed in R. }
$$

\section{Results}

\subsection{Effects of Volatile and Water Soluble Chemicals on Seed Germination}

Significant effects of the volatile chemicals released from A. herba-alba aerial parts were not observed on germination of $S$. vermiculata and L. spartum seeds (Table 1, Fig. B.1). However, the volatile chemicals released from the highest amount of A. herbaalba aerial parts inhibited germination of $P$. halepensis (Table 1, Fig. 1A). Furthermore, the volatile chemicals released from any amount of $A$. herba-alba aerial parts also inhibited the germination of its own seeds (Table 1, Fig. 1B).

The water soluble chemicals of $A$. herba-alba did not have a significant effect on the germination of S. vermiculata and L. spartum seeds (Table 1, Fig. B.2). On the other hand, germination of $P$. halepensis seeds differed significantly among treatments (Table 1). Specifically, the intermediate concentration of aqueous extract promoted seed germination compared to the highest concentration of aqueous extract. However, they did not differ significantly from seeds in the control (Figure 2A). With respect to 
germination of $A$. herba-alba seeds, there was an interaction between treatment and time of measurement (Table 1). Overall, the highest concentration of aqueous extract inhibited the germination of $A$. herba-alba seeds at all three times considered (d7, d14 and d21), whereas the lowest and intermediate concentrations only inhibited its germination from d7 (Fig. 2B).

The mixture of pure phenolic compounds (i.e., catechol, protocatechuic and vanillic acids) did not have a significant effect on the germination of $S$. vermiculata, $L$. spartum and P. halepensis seeds (Table 1, Figs. B.3 and 3A). However, the highest concentration of the mixture significantly inhibited germination of A. herba-alba seeds (Table 1, Fig. 3B).

\subsection{Effects of Volatile and Water Soluble Chemicals on Early Seedling Growth}

After 8 weeks, the volatile chemicals released from the intermediate and the highest amount of $A$. herba-alba aerial parts significantly reduced the root biomass of $S$. vermiculata seedlings $\left(F_{3,45}=11.96, \mathrm{p}<0.001\right)$, but non-significant effects were observed on the aerial biomass $\left(F_{3,45}=1.64, \mathrm{p}=0.19\right.$; Fig. 4$)$. Conversely, the volatile chemicals did not have a statistically significant effect on the root biomass L. spartum seedlings $\left(F_{3,56}=2.5, \mathrm{p}=0.07\right)$, but aerial biomass differed significantly among treatments $\left(F_{3,56}=3.84, \mathrm{p}=0.01\right)$. Specifically, aerial biomass was lower in $L$. spartum seedlings exposed to the volatile chemicals released from the intermediate amount of ground aerial parts than it was in those exposed to the lowest amount. However, they did not differ significantly from seedlings in the control (Fig. 4). In P. halepensis, aerial and root biomass were significantly higher in seedlings exposed to the highest amount of ground aerial parts of $A$. herba-alba than they were in seedlings in the control $\left(F_{3,49}=\right.$ 3.03, $\mathrm{p}=0.04$ and $F_{3,49}=5.12, \mathrm{p}=0.004$; Fig. 4). At all three treatments, the volatile 
331

332

333

334

335

336

337

338

339

340

341

342

343

344

345

346

347

348

349

350

351

352

353

chemicals of $A$. herba-alba aerial parts did not have a significant effect on the aerial $\left(F_{3,54}=1, \mathrm{p}=0.4\right)$ or the root biomass $\left(F_{3,51}=1.74, \mathrm{p}=0.17\right)$ of $A$. herba-alba seedlings (Fig. 4).

The effects of the aqueous extract of $A$. herba-alba on early seedling growth of S. vermiculata and A. herba-alba could not be quantified because mortality was very high and too few seedlings survived to the end of the bioassay (data not shown). The aerial biomass of L. spartum seedlings watered with the highest concentration of aqueous extract was significantly higher than the aerial biomass of seedlings in the control $\left(F_{3,55}=2.72, \mathrm{p}=0.05\right)$, but seedling root biomass did not differ significantly among the treatments $\left(F_{3,55}=2.11, \mathrm{p}=0.11 ;\right.$ Fig. 5$)$. In addition, both the aerial and root biomass were significantly higher in the seedlings of $P$. halepensis irrigated with either the intermediate or the highest concentration of aqueous extract than they were in the control seedlings $\left(F_{3,53}=4.16, \mathrm{p}=0.01\right.$ and $F_{3,52}=4.29, \mathrm{p}=0.009 ;$ Fig. 5).

\subsection{Volatile and Phenolic Constituents}

The GC-MS analysis allowed the identification of 49 volatile constituents, accounting for $92.58 \%$ of total VOCs (Table A.2). Among the identified compounds, oxygenated monoterpenes were the most abundant $(43.58 \%)$, followed by sesquiterpene hydrocarbons (37.24\%) and monoterpene hydrocarbons (10.9\%). Only a small amount of oxygenated sesquiterpenes were found (0.4\%). Specifically, 1,8 cineole (8.7\%), camphor $(6.9 \%)$ and cis-chrysanthenyl acetate $(14.9 \%)$ were found to be major constituents, accounting for two thirds of the oxygenated monoterpenes. The major sesquiterpene hydrocarbon was $\delta$-cadinene $(10.5 \%)$. $\alpha$-pinene $(3.25 \%)$ and $p$-cymene (2.6\%) accounted for over a half of the monoterpene hydrocarbons (Table A.2). 
$\left.1.15 \mathrm{mg} \mathrm{GAE} \mathrm{g}^{-1}\right)$ and stems $\left(36.15 \pm 0.48 \mathrm{mg} \mathrm{GAE} \mathrm{g}^{-1}\right)$ were approximately four and three times higher, respectively, than the TPC in the aqueous extract of $A$. herba-alba abundant. However, only three of the compounds could be identified and quantified in the aqueous extract: catechol, protocatechuic and vanillic acids (37.2, 9.4, and $2.2 \mathrm{mg} \mathrm{l}^{-1}$ respectively; Fig. 6).

\section{Discussion}

In this study we investigated the phytotoxic effects of the volatile and the water soluble chemicals produced by the shrub A. herba-alba on seed germination and early seedling growth of species that co-exist with $A$. herba-alba in natural semiarid plant communities, including A. herba-alba itself. Overall, our results showed that both the volatile and water soluble chemicals strongly inhibit the germination of A. herba-alba seeds, highlighting the autotoxic nature of this species. Previous studies also found autotoxic effects in A. herba-alba. Atoum et al. (2006) reported that exposure to aqueous extract obtained from $A$. herba-alba fruits might reduce initial elongation of $A$. herba-alba seedlings. In addition, it was found that water soluble chemicals can increase the mortality of $A$. herba-alba seedlings that emerge from the seed bank near $A$. herba-alba individuals (Arroyo et al., 2016). In a field study, Pueyo et al. (2016) found a pattern of segregation between adult $A$. herba-alba individuals and other adults,

377 found that although $A$. herba-alba seeds are dispersed in close proximity to the parent 
plants, only few seedlings are established under their canopy. Our results suggest that the volatile and the water soluble chemicals may drive autotoxicity of $A$. herba-alba during germination, which might lead to the observed pattern among A. herba-alba individuals in natural plant communities.

Autotoxicity has been observed in many crop systems (Singh et al., 1999), but also in natural environments such as Mediterranean forest of P. halepensis (Fernandez et al., 2008) and shrublands of Cistus ladanifer L. (Alías et al., 2006) and Cistus clusii Dunal (Foronda et al. in rev.). However, its ecological meaning is still not fully understood. It has been suggested that autotoxicity is the consequence of chemical defenses against herbivores and pathogens, or that it forces higher seed dispersal distances, favoring the colonization of new areas and avoiding inbreeding (Nilsen, 2003; Reigosa et al., 1999; Singh et al., 1999). Alternatively, given that individuals of the same species occupy a similar niche, a strong competition for resources might be expected to occur among them (Armas and Pugnaire, 2011). Therefore, in semiarid environments where resources are scarce (e.g., water), autotoxicity may arise as an adaptive advantage to regulate population density, diminishing self-recruitment near parent species by inhibiting or delaying germination until rainfall and, thereby, preventing intraspecific competition (Armas and Pugnaire, 2011; Friedman and Waller, 1985; Singh et al., 1999). Indeed, autotoxicity (or biochemical recognition sensu Renne et al., 2014) might be a common strategy to avoid competitive environments (Renne et al., 2014). Thus, autotoxicity might play a more important role than previously thought in determining the structure and dynamics of allelopathic shrub species in semiarid environments.

The composition of the essential oils of A. herba-alba, has been thoroughly investigated (Mohamed et al., 2010). Overall, oxygenated monoterpenes are often found 
403

404

405

406

407

408

409

410

411

412

413

414

415

416

417

418

419

420

421

422

423

424

425

426

427

to be the major constituents of the volatile fraction. In particular, the presence of $\alpha / \beta$ thujones, camphor, chrysanthenone and 1,8-cineole is commonly reported in essential oils of A. herba-alba (Bourgou et al., 2016; Mohamed et al., 2010; Salido et al., 2004; Younsi et al., 2016). In agreement with previous studies on the chemical composition of essential oils of A. herba-alba from Spain (Feuerstein et al., 1988; Salido et al., 2004), we found large amounts of 1,8 cineole and the absence of thujones. However, it was unusual the presence of nonanal. Many volatile constituents of $A$. herba-alba, such as $\alpha / \beta$-pinene, camphor and 1,8 cineole, has been reported as phytotoxic (Martino et al., 2010). However, the major volatile compound in our sample, cis-chrysanthenyl acetate (16.52\%), has not been reported as phytotoxic yet. Altogether, our results underscore the potential of $A$. herba-alba litter as a significant source of volatile phytotoxic compounds involved in its autotoxicity.

We also found an important total amount of phenolic compounds in the leaves and stems of $A$. herba-alba. Some studies have reported TPC values over $100 \mathrm{mg}$ GAE $\mathrm{g}^{-1}$ in A. herba-alba (Khlifi et al., 2013) but others have reported values that were lower than those in our study (Bourgou et al., 2016; Younsi et al., 2016). The variation in TPC among studies might be caused by differences in the plant organs used in the analyses, in the season of plant material collection, in climatic conditions in different regions and in the extraction procedure (Khlifi et al., 2013). Similarly, phenolic compounds including catechol, protocatechuic and vanillic acids were abundant in the aqueous extract of A. herba-alba. Protocatechuic and vanillic acids were already reported in $A$. herba-alba (Bourgou et al., 2016; Seddik et al., 2010). Our results showed that the pure phenolic mixture inhibited germination of $A$. herba-alba seeds, thus, suggesting that identified phenols might have contributed to the net autotoxic effect of the aqueous extract of A. herba-alba. However, autotoxic effects of the aqueous extract were 
apparently stronger than the effects of the mixture of pure phenols. On the one hand, there were other compounds in the aqueous extract of $A$. herba-alba that were unidentified, and might have contributed more to its autotoxicity than did the identified phenols. In this sense, it should be noted that the HPLC chromatogram at $280 \mathrm{~nm}$ indicated the presence of an extraordinarily abundant unidentified compound (peak 7, Fig. 3). Artemisia herba-alba aerial parts are known to contain other phenolic compounds such as chlorogenic, ferulic and caffeic acids, apigenin, luteolin and quercetin (Saleh et al., 1985; Seddik et al., 2010; Younsi et al., 2016). In addition, it could be possible that there were very small amounts of certain compounds belonging to other chemical classes (e.g., terpenes) in the aqueous extract (Weidenhamer et al., 1993), which might have also inhibitory effects. On the other hand, pure compounds in the mixture probably degrade faster than they do in the aqueous extract (Li et al., 2015).

On the other hand, our results showed limited phytotoxic effects of the volatile and water soluble chemicals produced by A. herba-alba on the germination of neighboring plant species. However, water soluble chemicals from A. herba-alba had positive effects on early seedling growth of $L$ spartum and P. halepensis. Low concentrations of phenolic acids such as those identified in the aqueous extract of $A$. herba-alba can induce stimulatory responses (Einhellig, 2004). Similarly, we found that the volatile chemicals of $A$. herba-alba had a positive effect on the early seedling growth of $P$. halepensis. In line with our results, Tsubo et al. (2012) found that exposure to low concentrations of the volatile chemicals released by Artemisia adamsii Besser stimulated photosynthetic rates in the grass Stipa krilovii Roshev., and, hence, its growth. It is likely that those species have some kind of tolerance to the allelochemicals produced by $A$. herba-alba. A long associational history between allelopathic plants (and their allelochemicals) and other coexisting species might lead some species to 
453

become adapted to its chemical neighbor (Ehlers and Thompson, 2004; Mallik and Pellissier, 2000). Our results suggest that in such cases a positive effect of volatile and water soluble chemicals on plant growth might be expected. In fact, it has been found that, in semiarid plant communities of the Middle Ebro Valley, A. herba-alba may act as a nurse plant facilitating the establishment of L. spartum and other perennial grasses regardless of its phytotoxicity (Arroyo et al., 2015; Pueyo et al., 2016). The ratio of susceptible and tolerant species will dictate the impacts of an allelopathic species at community level. Consequently, phytotoxic effects should be assessed from a multispecific perspective, including a number of target species covering several life forms and abundances in order to get reliable data on the impacts of an allelopathic species on plant community organization.

In conclusion, our study underscored that both the volatile and the water soluble chemicals might be involved in intra- and inter-specific interactions in semiarid environments. We found only limited phytotoxic effects of the volatile chemicals released from $A$. herba-alba on the germination of some neighboring plant species $(P$. halepensis) and generalized positive effects of the volatile and water soluble chemicals on early seedling growth. These findings suggested that target species selected in our study might have a certain tolerance to phytotoxic compounds of A. herba-alba because of co-evolution. On the other hand, both types of chemicals had strong autotoxic effects on the germination of $A$. herba-alba seeds. Furthermore, we found that phenolic compounds were abundant in the A. herba-alba aqueous extract. Our results emphasized that the identified phenolic compounds can be partially responsible for the autotoxicity of the aqueous extract, pointing out the potential role that these chemicals can play in semiarid environments. Further bioassays should use living donor plants and incorporate soil microorganisms in order to obtain more realistic evidences about the 
significance of allelopathy in natural conditions. In addition, more research is required

to fully understand the significance of autotoxicity in semiarid environments and its ecological meaning.

\section{Acknowledgements}

We especially thank to C. Gallet for her invaluable help with biochemical analyses,

M.L. Giner and P. Bravo for their field assistance, help with the experimental set up and data collection and J. Cosculluela ("Viveros Ejea") for kindly providing P. halepensis seeds used in bioassays. We are grateful to two anonymous reviewers for constructive criticism that significantly improved early version of this manuscript. This research was supported by the Spanish Ministry of Education, Culture and Sport (AP-2012-4126 grant to A.I.A., FPU Program) and by the Spanish Ministry of Economy and Competitiveness (CGL-2012-37508 and CGL-2016-80783-R projects).

\section{References}

Akula, R., Ravishankar, G.A., 2011. Influence of abiotic stress signals on secondary metabolites in plants. Plant Signal. Behav. 6, 1720-1731. doi:10.4161/psb.6.11.17613

Alías, J.C., Sosa, T., Escudero, J.C., Chaves, N., 2006. Autotoxicity against germination and seedling emergence in Cistus ladanifer L. Plant Soil 282, 327-332. doi:10.1007/s11104-005-6066-y

Araniti, F., Sorgonà, A., Lupini, A., Abenavoli, M.R., 2012. Screening of Mediterranean wild plant species for allelopathic activity and their use as bioherbicides. Allelopathy J. 29, 107-124.

Armas, C., Pugnaire, F.I., 2011. Belowground zone of influence in a tussock grass species. Acta Oecologica 37, 284-289. doi:10.1016/j.actao.2011.02.013

Arroyo, A.I., Pueyo, Y., Reiné, R., Giner, M.L., Alados, C.L., 2016. Effects of the allelopathic plant Artemisia herba-alba Asso on the soil seed bank of a semi-arid plant community. J. Plant Ecol. rtw120. doi:10.1093/jpe/rtw120

Arroyo, A.I., Pueyo, Y., Saiz, H., Alados, C.L., 2015. Plant-plant interactions as a mechanism structuring plant diversity in a Mediterranean semi-arid ecosystem. Ecol. Evol. 5, 5305-5317. doi:10.1002/ece3.1770

Atoum, M., Al-Charchafchi, F., Modallal, N., 2006. Biological activity and antimutagencity of water soluble phytotoxins from Artemisia herba alba Asso. Pak. J. Biol. Sci. 9, 1774-1778. doi:10.3923/pjbs.2006.1774.1778 
Bautista, I., Boscaiu, M., Lidón, A., Llinares, J.V., Lull, C., Donat, M.P., Mayoral, O., Vicente, O., 2016. Environmentally induced changes in antioxidant phenolic compounds levels in wild plants. Acta Physiol. Plant. 38. doi:10.1007/s11738015-2025-2

Bourgou, S., Tammar, S., Salem, N., Mkadmini, K., Msaada, K., 2016. Phenolic composition, essential oil, and antioxidant activity in the aerial part of Artemisia herba-alba from several provenances: a comparative study. Int. J. Food Prop. 19, 549-563. doi:10.1080/10942912.2015.1040495

Callaway, R.M., 2007. Direct Mechanisms for Facilitation, in: Callaway, R.M. (Ed.), Positive Interactions and Interdependence in Plant Communities. Springer Netherlands, Dordrecht, pp. 15-116.

Castaldi, S., Carfora, A., Fiorentino, A., Natale, A., Messere, A., Miglietta, F., Cotrufo, M.F., 2009. Inhibition of net nitrification activity in a Mediterranean woodland: possible role of chemicals produced by Arbutus unedo. Plant Soil 315, 273-283. doi:10.1007/s11104-008-9750-x

Chen, S., Xiao, S., Callaway, R.M., 2012. Light intensity alters the allelopathic effects of an exotic invader. Plant Ecol. Divers. 5, 521-526. doi:10.1080/17550874.2012.736548

Chou, C.-H., 1999. Roles of allelopathy in plant biodiversity and sustainable agriculture. Crit. Rev. Plant Sci. 18, 609-636. doi:10.1080/07352689991309414

Ehlers, B.K., Thompson, J., 2004. Do co-occurring plant species adapt to one another? The response of Bromus erectus to the presence of different Thymus vulgaris chemotypes. Oecologia 141, 511-518. doi:10.1007/s00442-004-1663-7

Einhellig, F.A., 2004. Mode of allelochemical action of phenolic compounds, in: Macias, F.A., Galindo, J.C.G., Molinillo, J.M.G., Cutler, H.G. (Eds.), Allelopathy: Chemistry and Mode of Action of Allelochemicals. CRC Press, Boca Raton, FL, pp. 217-238.

Fernandez, C., Voiriot, S., Mévy, J.-P., Vila, B., Ormeño, E., Dupouyet, S., BousquetMélou, A., 2008. Regeneration failure of Pinus halepensis Mill.: The role of autotoxicity and some abiotic environmental parameters. For. Ecol. Manag. 255, 2928-2936. doi:10.1016/j.foreco.2008.01.072

Feuerstein, I., Danin, A., Segal, R., 1988. Constitution of the essential oil from an Artemisia herba-alba population of Spain. Phytochemistry 27, 433-434. doi:10.1016/0031-9422(88)83114-5

Friedjung, A., Choudhary, S.P., Dudai, N., Rachmilevitch, S., 2013. Physiological conjunction of allelochemicals and desert plants. PLoS ONE 8, e81580. doi:10.1371/journal.pone.0081580

Friedman, J., Orshan, G., 1975. The distribution, emergence and survival of seedlings of Artemisia herba-alba Asso in the Negev desert of Israel in relation to distance from the adult plants. J. Ecol. 63, 627. doi:10.2307/2258740

Friedman, J., Orshan, G., Ziger-Cfir, Y., 1977. Suppression of annuals by Artemisia herba-alba in the Negev desert of Israel. J. Ecol. 65, 413-426. doi:10.2307/2259491

Friedman, J., Waller, G.R., 1985. Allelopathy and autotoxicity. Trends Biochem. Sci. 10, 47-50. doi:10.1016/0968-0004(85)90224-5

Hättenschwiler, S., Vitousek, P.M., 2000. The role of polyphenols in terrestrial ecosystem nutrient cycling. Trends Ecol. Evol. 15, 238-243. doi:10.1016/S0169-5347(00)01861-9 
Herranz, J.M., Ferrandis, P., Copete, M.A., Duro, E.M., Zalacaín, A., 2006. Effect of allelopathic compounds produced by Cistus ladanifer on germination of 20 Mediterranean taxa. Plant Ecol. 184, 259-272. doi:10.1007/s11258-005-9071-6 Inderjit, Mallik, A.U., 1997. Effect of phenolic compounds on selected soil properties. For. Ecol. Manag. 92, 11-18. doi:10.1016/S0378-1127(96)03957-6

Khlifi, D., Sghaier, R.M., Amouri, S., Laouini, D., Hamdi, M., Bouajila, J., 2013. Composition and anti-oxidant, anti-cancer and anti-inflammatory activities of Artemisia herba-alba, Ruta chalpensis L. and Peganum harmala L. Food Chem. Toxicol. 55, 202-208. doi:10.1016/j.fct.2013.01.004

Li, J., Zhang, Q., Hu, W., Yang, X., He, H., 2015. Stability of phenolic acids and the effect on weed control activity. J. Korean Soc. Appl. Biol. Chem. 58, 919-926. doi:10.1007/s13765-015-0124-9

Li, Z.H., Wang, Q., Ruan, X., Pan, C.-D., Jiang, D.-A., 2010. Phenolics and plant allelopathy. Molecules 15, 8933-8952. doi:10.3390/molecules15128933

Ludwig, J.A., Wilcox, B.P., Breshears, D.D., Tongway, D.J., Imeson, A.C., 2005. Vegetation patches and runoff-erosion as interacting ecohydrological process in semiarid landscapes. Ecology 86, 288-297. doi:10.1890/03-0569

Mallik, A.U., Pellissier, F., 2000. Effects of Vaccinium myrtillus on spruce regeneration: testing the notion of coevolutionary significance of allelopathy. J. Chem. Ecol. 26, 2197-2209. doi:10.1023/A:1005528701927

Martino, L.D., Mancini, E., Almeida, L.F.R. de, Feo, V.D., 2010. The Antigerminative Activity of Twenty-Seven Monoterpenes. Molecules 15, 6630-6637. doi:10.3390/molecules15096630

Mohamed, A.E.-H.H., El-Sayed, M.A., Hegazy, M.E., Helaly, S.E., Esmail, A.M., Mohamed, N.S., 2010. Chemical constituents and biological activities of Artemisia herba-alba. Rec. Nat. Prod. 4, 1-25.

Muscolo, A., Sidari, M., 2010. Source of soil phenolic compounds, in: Muscolo, A., Sidari, M. (Eds.), Soil Phenols. Nova Science Publishers, Inc., New York, NY, pp. $1-30$.

Nilsen, E.T., 2003. Ecological relevance of allelopathy: some considerations related to Mediterranean, subtropical, temperate, and boreal forest shrubs, in: Mallik, A.U., Inderjit (Eds.), Chemical Ecology of Plants: Allelopathy in Aquatic and Terrestrial Ecosystems. Birkhäuser Basel, Basel, pp. 109-129. doi:10.1007/9783-0348-8109-8_8

Pedrol, N., González, L., Reigosa, M.J., 2006. Allelopathy and abiotic stress, in: Reigosa, M.J., Pedrol, N., González, L. (Eds.), Allelopathy. Springer Netherlands, Dordrecht, pp. 171-209.

Pueyo, Y., Moret-Fernández, D., Arroyo, A.I., de Frutos, A., Kéfi, S., Saiz, H., Charte, R., Giner, M. de la L., Alados, C.L., 2016. Plant nurse effects rely on combined hydrological and ecological components in a semiarid ecosystem. Ecosphere 7, e01514. doi:10.1002/ecs2.1514

Reigosa, M.J., Sánchez-Moreiras, A., González, L., 1999. Ecophysiological approach in allelopathy. Crit. Rev. Plant Sci. 18, 577-608. doi:10.1080/07352689991309405

Renne, I.J., Sinn, B.T., Shook, G.W., Sedlacko, D.M., Dull, J.R., Villarreal, D., Hierro, J.L., 2014. Eavesdropping in plants: delayed germination via biochemical recognition. J. Ecol. 102, 86-94. doi:10.1111/1365-2745.12189

Rice, E.L., 1984. Allelopathy (Second Edition), Physiological Ecology. Academic Press, San Diego.

Ruan, X., Li, Z.-H., Wang, Q., Pan, C.-D., Jiang, D.-A., Wang, G.G., 2011. Autotoxicity and allelopathy of 3,4-dihydroxyacetophenone isolated from Picea 
schrenkiana needles. Molecules 16, 8874-8893. doi:10.3390/molecules 16108874

Saleh, N.A.M., El-Negoumy, S.I., Abd-Alla, M.F., Abou-Zaid, M.M., Dellamonica, G., Chopin, J., 1985. Flavonoid glycosides of Artemisia monosperma and A. herbaalba. Phytochemistry 24, 201-203. doi:10.1016/S0031-9422(00)80845-6

Salido, S., Valenzuela, L.R., Altarejos, J., Nogueras, M., Sánchez, A., Cano, E., 2004. Composition and infraspecific variability of Artemisia herba-alba from southern Spain. Biochem. Syst. Ecol. 32, 265-277. doi:10.1016/j.bse.2003.09.002

Seddik, K., Nadjet, I., Daoud, B.A.H., Lekhmici, A., 2010. Antioxidant and antibacterial activities of extracts from Artemisia herba alba Asso. leaves and some phenolic compounds. J. Med. Plants Res. 4, 1273-1280. doi:10.5897/JMPR09.379

Singh, H.P., Batish, D.R., Kohli, R.K., 1999. Autotoxicity: concept, organisms, and ecological significance. Crit. Rev. Plant Sci. 18, 757-772. doi:10.1080/07352689991309478

Singleton, V.L., Rossi, J.A., 1965. Colorimetry of total phenolics with phosphomolybdic-phosphotungstic acid reagents. Am. J. Enol. Vitic. 16, 144158.

Tsubo, M., Nishihara, E., Nakamatsu, K., Cheng, Y., Shinoda, M., 2012. Plant volatiles inhibit restoration of plant species communities in dry grassland. Basic Appl. Ecol. 13, 76-84. doi:10.1016/j.baae.2011.11.005

van der Putten, W.H., 2009. A multitrophic perspective on functioning and evolution of facilitation in plant communities. J. Ecol. 97, 1131-1138. doi:10.1111/j.13652745.2009.01561.x

Varela, M.C., Arslan, I., Reginato, M.A., Cenzano, A.M., Luna, M.V., 2016. Phenolic compounds as indicators of drought resistance in shrubs from Patagonian shrublands (Argentina). Plant Physiol. Biochem. 104, 81-91. doi:10.1016/j.plaphy.2016.03.014

Wang, Jinli, Li, X., Zhang, J., Yao, T., Wei, D., Wang, Y., Wang, Jingguo, 2012. Effect of root exudates on beneficial microorganisms - evidence from a continuous soybean monoculture. Plant Ecol. 213, 1883-1892. doi:10.1007/s11258-0120088-3

Weidenhamer, J.D., Macias, F.A., Fischer, N.H., Williamson, G.B., 1993. Just how insoluble are monoterpenes? J. Chem. Ecol. 19, 1799-1807. doi:10.1007/BF00982309

Younsi, F., Trimech, R., Boulila, A., Ezzine, O., Dhahri, S., Boussaid, M., Messaoud, C., 2016. Essential oil and phenolic compounds of Artemisia herba-alba (Asso.): composition, antioxidant, antiacetylcholinesterase, and antibacterial activities. Int. J. Food Prop. 19, 1425-1438. doi:10.1080/10942912.2015.1079789 


\begin{tabular}{|c|c|c|c|c|c|c|c|c|c|c|c|c|c|}
\hline \multirow[b]{2}{*}{ Species } & \multirow[b]{2}{*}{ Variable } & \multicolumn{4}{|c|}{ Volatile chemicals } & \multicolumn{4}{|c|}{ Aqueous extract } & \multicolumn{4}{|c|}{ Phenolic mixture } \\
\hline & & Res. Deviance & $\chi^{2}$ & df & $p$-value & Res. Deviance & $\chi^{2}$ & df & $p$-value & Res. Deviance & $\chi^{2}$ & df & $p$-value \\
\hline \multicolumn{14}{|l|}{ S. vermiculata } \\
\hline & Treatment & 223.37 & 7.43 & 3 & 0.06 & 257.58 & 2.17 & 3 & 0.54 & 253.28 & 2.07 & 3 & 0.56 \\
\hline & Time & 230.79 & 0.01 & 1 & 0.92 & 259.64 & 0.11 & 1 & 0.74 & 255.23 & 0.11 & 1 & 0.73 \\
\hline & Treatment:Time & 223.34 & 0.02 & 3 & 0.99 & 257.30 & 0.17 & 3 & 0.98 & 253.12 & 0.05 & 3 & 0.99 \\
\hline \multicolumn{14}{|l|}{ L. spartum } \\
\hline & Treatment & 463.05 & 1.16 & 3 & 0.76 & 336.93 & 0.87 & 3 & 0.83 & 313.55 & 1.02 & 3 & 0.80 \\
\hline & Time & 281.52 & 182.7 & 2 & $<0.001$ & 289.34 & 48.46 & 2 & $<0.001$ & 271.01 & 43.56 & 2 & $<0.001$ \\
\hline & Treatment:Time & 273.76 & 6.62 & 6 & 0.36 & 281.11 & 7.37 & 6 & 0.29 & 267.41 & 2.62 & 6 & 0.85 \\
\hline \multicolumn{14}{|l|}{ P. halepensis } \\
\hline & Treatment & 849.99 & 22.02 & 3 & $<0.001$ & 652.20 & 11.66 & 3 & $<0.01$ & 369.20 & 6.95 & 3 & 0.07 \\
\hline & Time & 355.90 & 516.12 & 2 & $<0.001$ & 405.91 & 257.94 & 2 & $<0.001$ & 300.26 & 75.89 & 2 & $<0.001$ \\
\hline & Treatment:Time & 330.68 & 5.23 & 6 & 0.51 & 385.94 & 8.26 & 6 & 0.22 & 290.31 & 3.07 & 6 & 0.80 \\
\hline \multicolumn{14}{|l|}{ A. herba-alba } \\
\hline & Treatment & 263.91 & 27.27 & 3 & $<0.001$ & 566.72 & 37.53 & 3 & $<0.001$ & 552.56 & 15.81 & 3 & $<0.01$ \\
\hline & Time & 271.60 & 19.58 & 2 & $<0.001$ & 382.80 & 221.45 & 2 & $<0.001$ & 343.23 & 225.14 & 2 & $<0.001$ \\
\hline & Treatment:Time & 243.74 & 0.57 & 6 & 0.99 & 329.77 & 15.07 & 6 & 0.02 & 317.60 & 9.53 & 6 & 0.15 \\
\hline
\end{tabular}


652

653

654

655

656

657

658

659

660

661

662

663

664

665

666

667

668

669

670

671

672

673

\section{Figure legends}

Fig. 1 Germination (mean $\% \pm \mathrm{SE}$ ) of A) P. halepensis and B) A. herba-alba seeds exposed to volatile chemicals released from $0.05,0.1$ and $0.5 \mathrm{~g}$ of ground A. herba-alba aerial parts. Different letters indicate significant differences among treatments (Tukey's HSD test)

Fig. 2 Germination (mean $\% \pm \mathrm{SE}$ ) of A) P. halepensis and B) A. herba-alba seeds moistened with $5 \mathrm{ml}$ of $A$. herba-alba aqueous extract diluted to $0.5,2$ and $5 \mathrm{~g}^{-1}$. Different letters indicate significant differences among treatments (Tukey's HSD test)

Fig. 3 Germination (mean $\% \pm$ SE) of A) P. halepensis and B) A. herba-alba seeds moistened with $5 \mathrm{ml}$ of a mixture of catechol, protocatechuic and vanillic acids. At each treatment, the concentration of each compound was the same as the concentration of such compounds in either $0.5,2$ or $5 \mathrm{~g} \mathrm{l}^{-1}$ of $A$. herba-alba aqueous extract. Different letters indicate significant differences among treatments (Tukey's HSD test)

Fig. 4 Dry biomass (mean \pm SE) of aerial parts (dark grey) and roots (light grey) of seedlings of S. vermiculata, L. spartum, P. halepensis and A. herba-alba (from left to right) after two months of exposure to either $0.05,0.17$ or $0.5 \mathrm{~g}$ per seedling of ground A. herba-alba aerial parts. Different letters among treatments indicate significant differences (Tukey's HSD test)

Fig. 5 Dry biomass (mean \pm SE) of aerial parts (dark grey) and roots (light grey) of seedlings of L. spartum (left side) and P. halepensis (right side) after two months of watering with either $0.5,2$ or $5 \mathrm{~g}^{-1}$ of aqueous extract of A. herba-alba. Different letters among treatments indicate significant differences (Tukey's HSD test) 
674 Fig. 6 HPLC profile of $A$. herba-alba aqueous extract. Numbered peaks indicate the 675 different phenolic compounds detected in aqueous extract at $280 \mathrm{~nm}$. Only three 676 compounds could be identified by comparing retention time, absorption spectrum and 677 the peak area to those of a standard 


\section{$679 \quad$ Fig. 1}
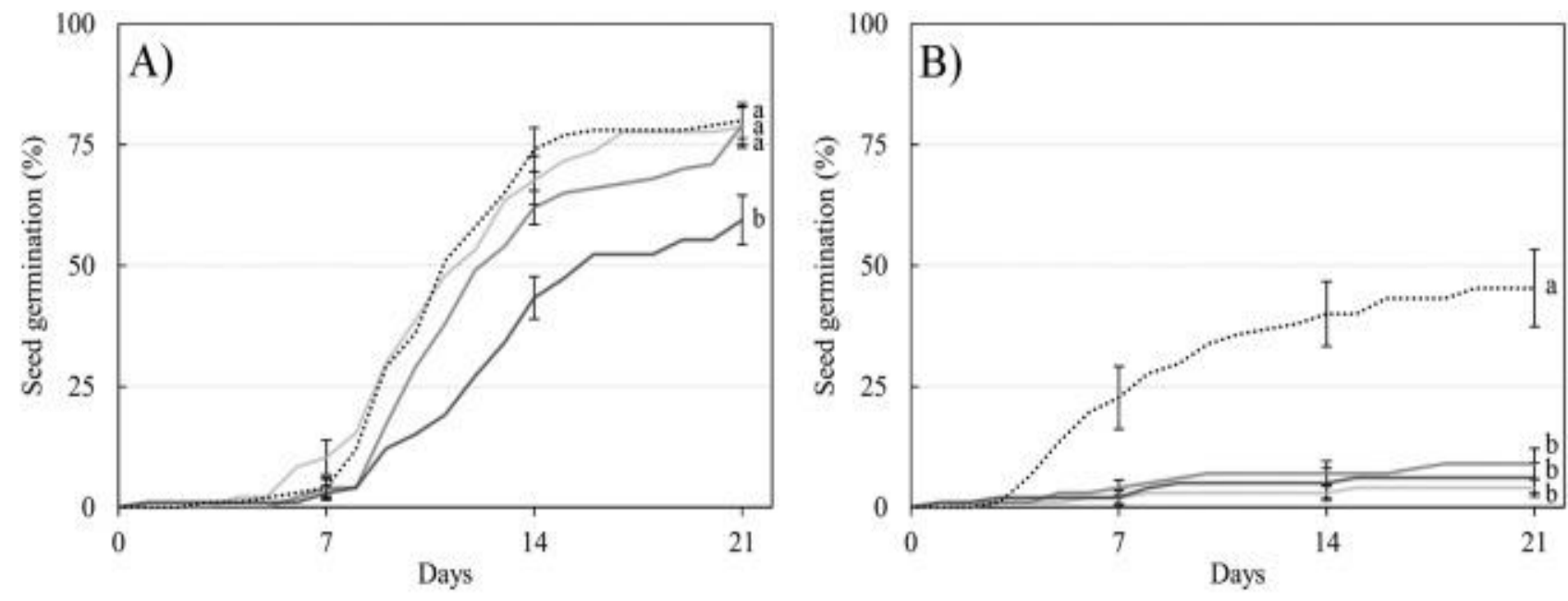

680

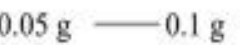

$0.5 \mathrm{~g}$........ Control

681

682 Fig. 2
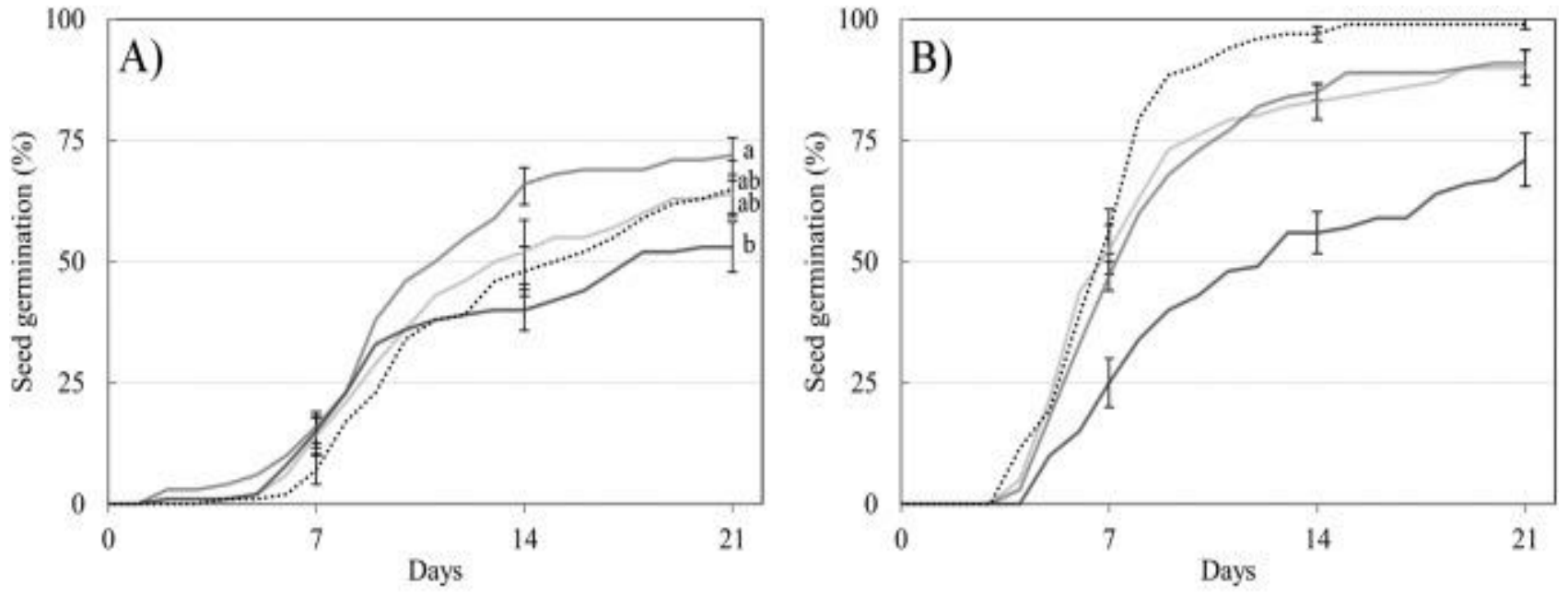

683

$-0.5 \mathrm{~g} / 1-2 \mathrm{~g} / \mathrm{l}-5 \mathrm{~g} / \mathrm{l}$........ Control

684

685

686 
Fig. 3
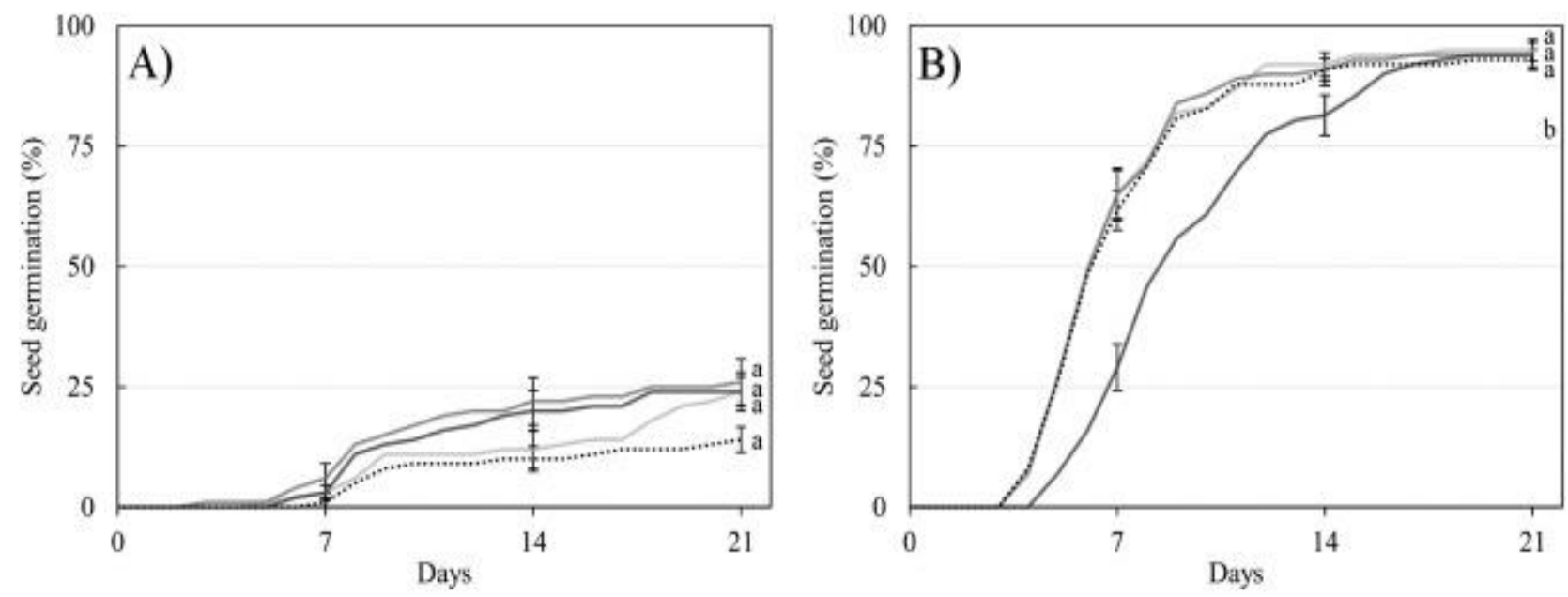

688

$-0.5 \mathrm{~g} / 1-2 \mathrm{~g} / \mathrm{l}-5 \mathrm{~g} / \mathrm{l} \cdots \cdots \cdot \ldots$. Control

689 Fig. 4

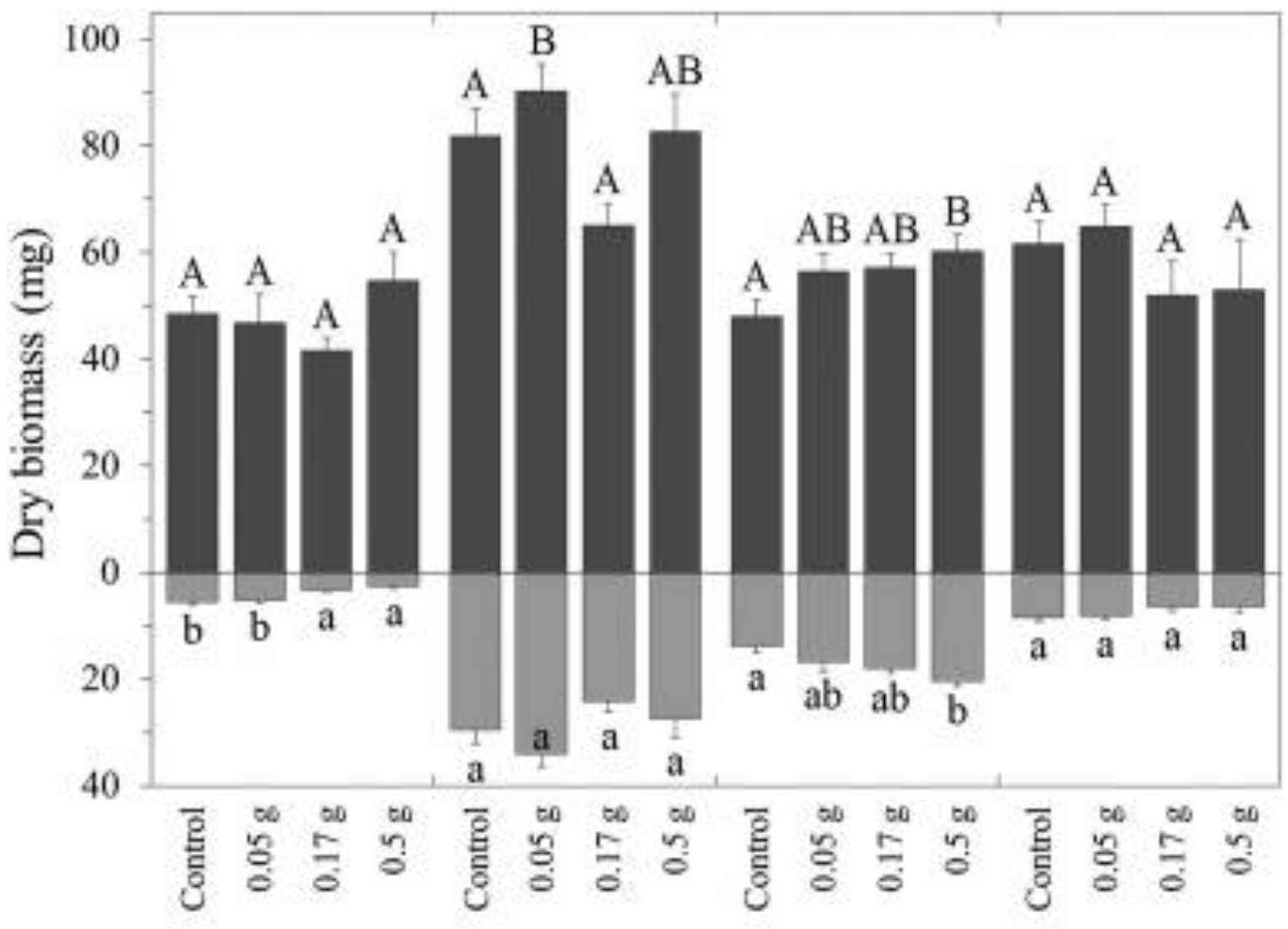

S. vermiculata L. spartum P.halepensis A.herba-alba

Volatile chemicals 
691 Fig. 5

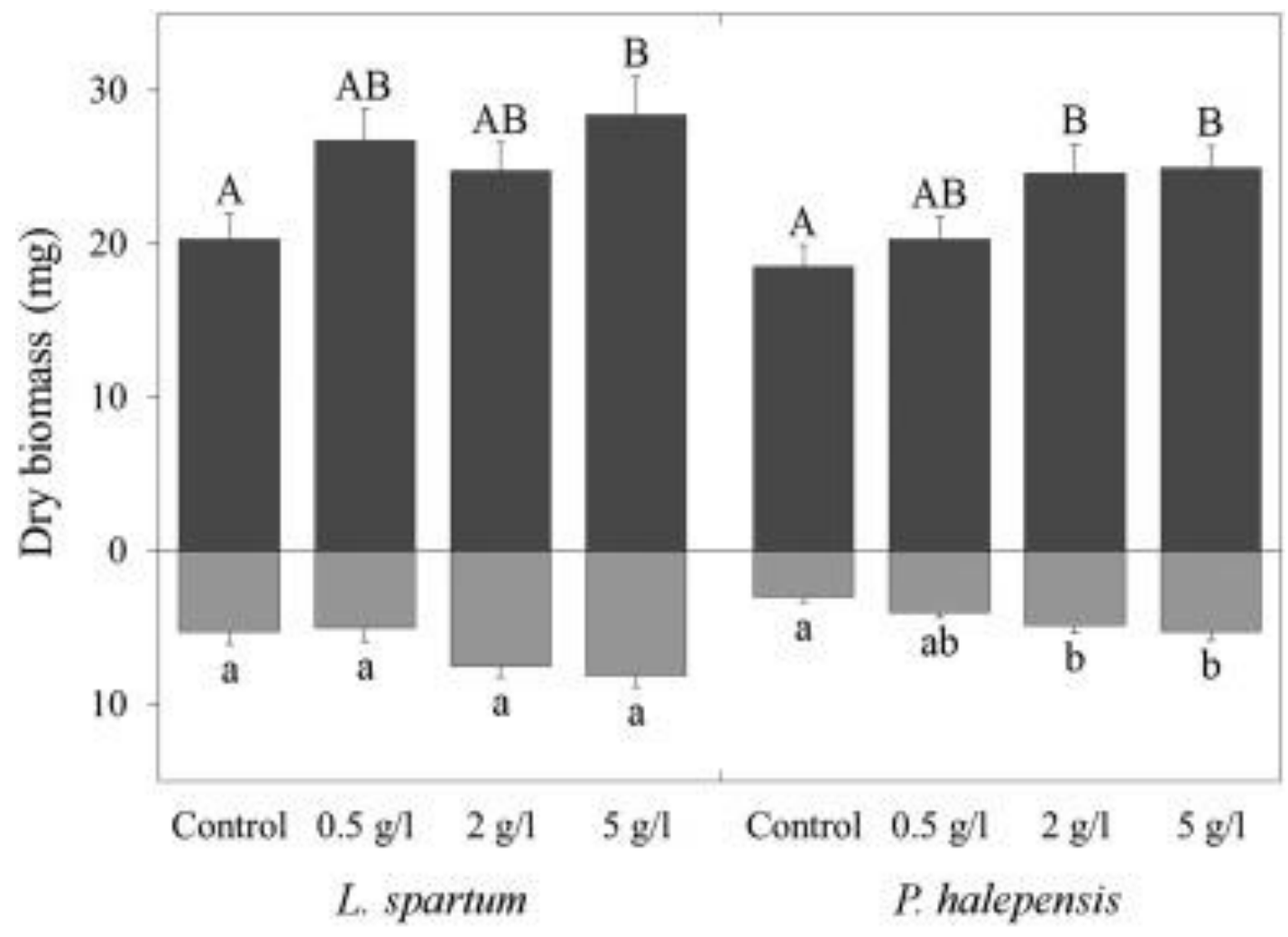

692

Aqueous extract

693 Fig. 6

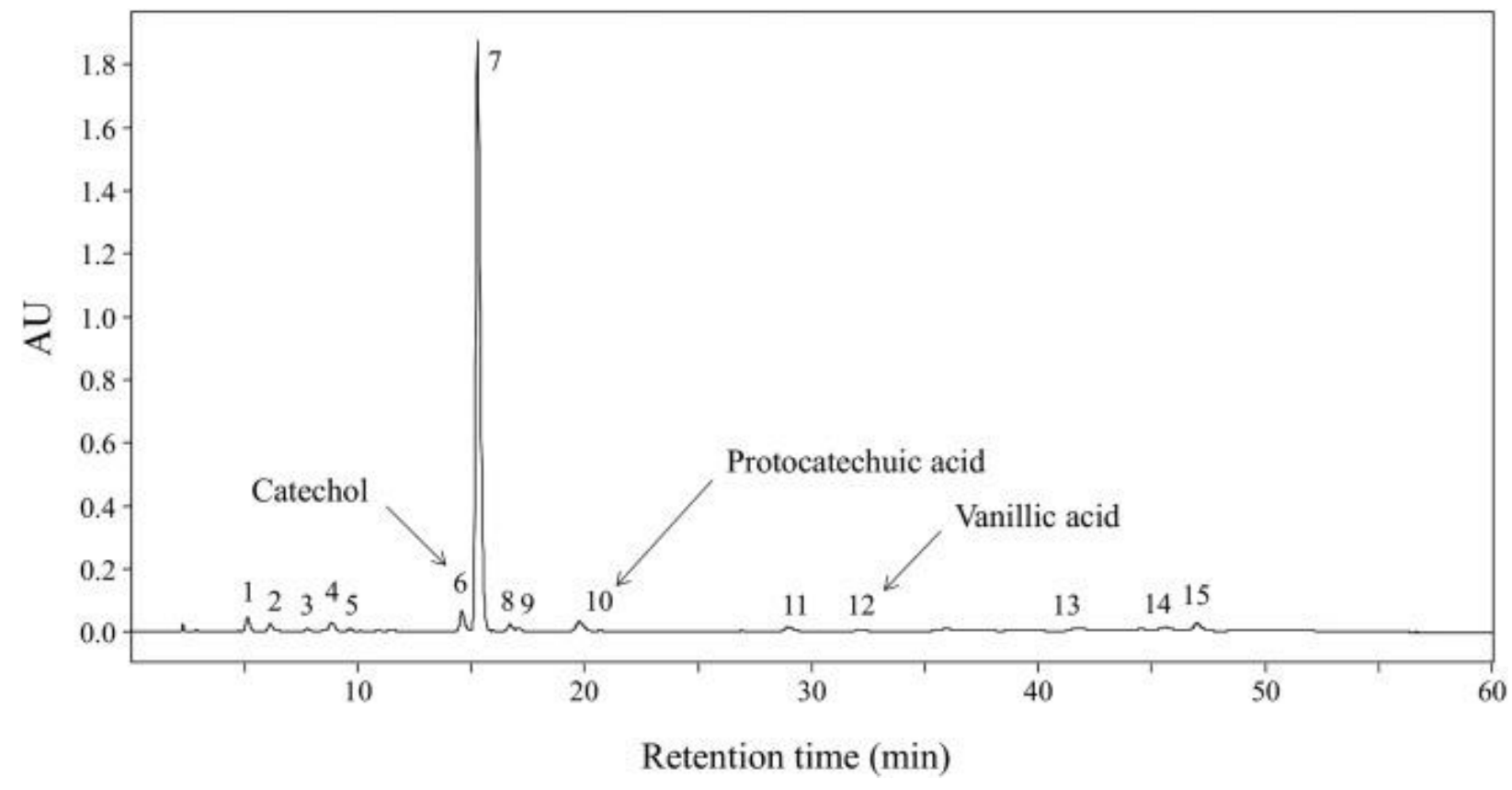

